\title{
Emergency department buprenorphine/naloxone: What we can achieve with system-level support and local champions
}

\author{
Jessica Moe, MD, MA, MSc ${ }^{* \dagger \neq}$; Jane A. Buxton, MBBS, MHSc ${ }^{\ddagger \S}$
}

This month's article by Dr. McLane et al. ${ }^{1}$ demonstrates the potential impact of emergency department (ED)-led initiatives, supported at a system level, to optimize care for patients with opioid use disorder, some of the most at-risk persons who pass through our ED doors. The authors report on a multi-site ED buprenorphine/naloxone program driven by the mandate of a province-wide clinical network. The intervention was guided by a highlevel conceptual framework and piloted at three ED sites. Clinical champions brought together multidisciplinary teams to develop site-specific solutions, which were adapted to local resources, capacity, and patient needs. The results reported herein are notable: intervention sites increased their rate of buprenorphine/naloxone dispensation from 0.25 to 5.9 visits per month preand post-intervention. Among patients provided buprenorphine/naloxone, $74.4 \%$ continued to fill opioid agonist therapy prescriptions at 60 days, determined by provincial pharmacy records. As emergency physicians, we interact with patients during a transient moment in their personal and medical journeys, and are often left wondering what happened to the individuals we have seen. If anything, the results of this study make one thing abundantly clear: when we identify a patient at risk for opioid overdose and offer them buprenorphine/naloxone, we are making a difference.

As much as this study provides a compelling argument for the imperative of offering buprenorphine/naloxone from the ED, its design and findings raise questions that require reflection to better understand the generalizability of results. The comparatively high retention rate $^{2}$ (a similar study reported a $25 \%$ six-month retention after buprenorphine/naloxone initiation) begs for a more in-depth exploration of patient and physician characteristics. Patients were enrolled at the discretion of treating physicians: inclusion was predicated on clinician suspicion of opioid use disorder and patient willingness to engage. Proportionally, $51.1 \%$ of intervention patients were female, compared with $35 \%$ in the non-intervention comparison group, suggesting a potential selection bias. Who were the patients who engaged, and who were those who declined (not captured by the current analysis)? Who were the physicians who provided buprenorphine/naloxone? What were the included patients' comorbidities, socioeconomic situations, prior experiences with healthcare, and motivators for engaging? In short, what made these patients so likely to be successful?

Another important question is, who are the patients we are trying to reach? The majority of the patients who received buprenorphine/naloxone in the current study had presented for complaints directly related to opioid use $(80.3 \%)$, which likely reflects the discretionary nature of the offered intervention. It makes sense to offer patients buprenorphine/naloxone when they present with opioid intoxication or withdrawal, and, as the study results indicate, this is an important window to offer interventions. Patients may be open to considering treatment after a harrowing intoxication experience or while experiencing the extreme discomfort of

From the * Department of Emergency Medicine, University of British Columbia, Vancouver; ${ }^{\dagger}$ Department of Emergency Medicine, Vancouver General Hospital, Vancouver, BC; ${ }^{\ddagger}$ British Columbia Centre for Disease Control, Vancouver, BC; and the ${ }^{\S}$ School of Population and Public Health, University of British Columbia, Vancouver, BC.

Correspondence to: Dr. Jessica Moe, Department of Emergency Medicine, Vancouver General Hospital, 920 West 10th Avenue, Vancouver, BC V5Z 1M9; Email: jessica.moe@ubc.ca.

(c) Canadian Association of Emergency Physicians 2020

CJEM 2020;22(6):735-737

DOI 10.1017/cem.2020.470 
withdrawal. However, patients presenting with opioidrelated complaints represent the very tip of the iceberg of individuals presenting to EDs who could benefit from buprenorphine/naloxone. Provincial public health data from British Columbia demonstrate that 54\% of persons who experienced an overdose visited an ED in the year prior to the overdose event, and a minority of their visits were substance-use-related. ${ }^{3} \mathrm{~A}$ seminal ED buprenorphine/naloxone study employed universal screening to identify eligible candidates; a mere $8.8 \%$ of enrolled patients had presented with an overdose. ${ }^{4}$ This evidence demonstrates that the denominator of ED patients with opioid use disorder who we could identify and potentially impact is far larger than those presenting with opioid intoxication or withdrawal. Developing and implementing evidence-based methods to identify patients with opioid use disorder who are at-risk for an overdose and likely to benefit from a targeted intervention ${ }^{5}$ (e.g., integrated screening or in situ peer navigators) remain a challenging but important task.

As individual Canadian EDs consider creating sitespecific buprenorphine/naloxone programs, it will be essential to consider an integral harm reduction principle: the importance of meeting people where they are at. To do so in the ED context, we need to understand the realities of the population we are trying to reach. How, when, and why do they come to the ED? What are their needs? What barriers do they face? To design effective programs with maximal impact, we need to approach this subject with flexibility, meaningfully involve people with lived and living experience with substance and opioid use, and explore options that could work across the diverse spectrum of patients whom we see and treat.

Identifying ED patients with opioid use disorder and mitigating their risk is of urgent importance, more so now than ever. Overdose rates in many places in Canada have spiked since the 2019 coronavirus disease pandemic began. British Columbia recorded 175 overdose deaths in June 2020, a 130\% increase compared with June 2019. ${ }^{6}$ We know that many people who come through our ED doors are at risk for an overdose: their ED visit may be a fleeting opportunity to identify them and prevent an overdose death. With a median age of 34 years among the included patients described by McLane et al., not only are emergency clinicians in a position to save lives, but furthermore to impact individuals who have the potential for long and productive lives. Significantly, 5 to $6 \%$ of patients who present to EDs following overdose die within one year, and one fifth of these deaths will occur within one month. ${ }^{7}$ Compare that to a $2.1 \%$ one-year mortality among patients over 40 years presenting for chest pain, ${ }^{8}$ or a $3.4 \%$ oneyear mortality among patients admitted to trauma centres $^{9}$ : both are conditions to which we pour resources, develop clinical pathways, and ensure comprehensive follow-up plans to improve outcomes. We must regard offering buprenorphine/naloxone, take-home naloxone kits, addictions follow-up, and harm reduction counselling as our duty, not a nicety or an option. This study provides an example of the importance of physician leadership and system-level support in enabling the implementation of ED buprenorphine/naloxone programs. Indeed, our care for patients with opioid use disorder will constitute some of the most impactful and career-defining interactions we are likely to make as emergency physicians.

Keywords: Buprenorphine, buprenorphine, naloxone drug combination, emergency medicine, opioid epidemic, public health

Competing interests: None declared.

\section{REFERENCES}

1. McLane, et al. Multi-site intervention to improve emergency department care for patients who live with opioid use disorder: A quantitative evaluation. C7EM 2020;22(6):784-792.

2. Hu T, Snider-Adler M, Nijmeh L, Pyle A. Buprenorphine/ naloxone induction in a Canadian emergency department with rapid access to community-based addictions providers. CFEM 2019;21(4):492-8.

3. Otterstatter MC, Crabtree A, Dobrer S, et al. Patterns of health care utilization among people who overdosed from illegal drugs: a descriptive analysis using the $\mathrm{BC}$ provincial overdose cohort. Health promotion and chronic disease prevention in Canada: research, policy and practice 2018;38(9): 328-38.

4. D'Onofrio G, O'Connor PG, Pantalon MV, et al. Emergency department-initiated buprenorphine/naloxone treatment for opioid dependence: a randomized clinical trial. $7 A M A$ 2015;313(16):1636.

5. Goedel WC, Marshall BDL, Samuels EA, et al. Randomised clinical trial of an emergency department-based peer recovery support intervention to increase treatment uptake and reduce recurrent overdose among individuals at high risk for opioid overdose: study protocol for the navigator trial. BMF Open 2019;9(11):e032052.

6. British Columbia Coroners Service. Illicit drug toxicity deaths in BC: January 1, 2010-June 30, 2020; 2020. Available at: https://www2.gov.bc.ca/assets/gov/birth-adoption-death- 
marriage-and-divorce/deaths/coroners-service/statistical/illicitdrug.pdf (accessed August 16, 2020).

7. Weiner SG, Baker O, Bernson D, Schuur JD. One-year mortality of patients after emergency department treatment for nonfatal opioid overdose. Ann Emerg Med 2020;75(1): $13-7$.
8. Ko DT, Dattani ND, Austin PC, et al. Emergency department volume and outcomes for patients after chest pain assessment. Circ Cardiovasc Qual Outcomes 2018;11(11): $\mathrm{e} 004683$.

9. Dutton RP, Stansbury LG, Leone S, et al. Trauma mortality in mature trauma systems: are we doing better? An analysis of trauma mortality patterns, 1997-2008. 7 Trauma 2010;69(3):620-6. 